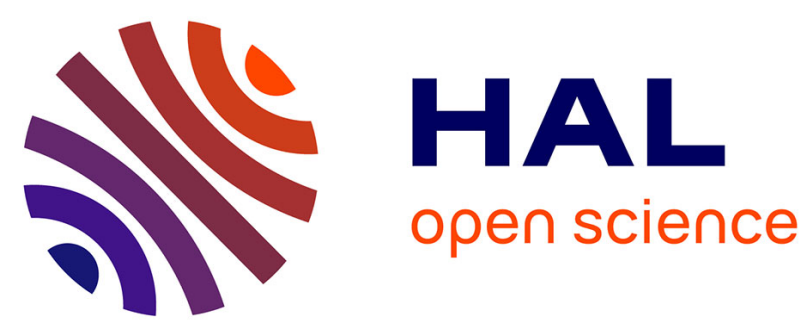

\title{
A model-based approach for statistical assessment of detection and localization performance of guided wave-based imaging techniques
}

Jérémy Moriot, Nicolas Quaegebeur, Alain Le Duff, Patrice Masson

\section{- To cite this version:}

Jérémy Moriot, Nicolas Quaegebeur, Alain Le Duff, Patrice Masson. A model-based approach for statistical assessment of detection and localization performance of guided wave-based imaging techniques. Structural Health Monitoring, 2017, 10.1177/1475921717744679 . hal-01656780

\section{HAL Id: hal-01656780 \\ https://hal.science/hal-01656780}

Submitted on 8 Feb 2018

HAL is a multi-disciplinary open access archive for the deposit and dissemination of scientific research documents, whether they are published or not. The documents may come from teaching and research institutions in France or abroad, or from public or private research centers.
L'archive ouverte pluridisciplinaire HAL, est destinée au dépôt et à la diffusion de documents scientifiques de niveau recherche, publiés ou non, émanant des établissements d'enseignement et de recherche français ou étrangers, des laboratoires publics ou privés. 


\title{
A model-based approach for statistical assessment of detection and localization performance of guided wave-based imaging techniques
}

\author{
Jérémy Moriot (1,2,3)* Nicolas Quaegebeur(2), Alain Le Duff(1,3), and Patrice Masson(2)
}

Keywords: Structural Health Monitoring, performance indicators, probability of detection, probability of localization, MAPOD, guided wave-based imaging.

\begin{abstract}
This paper aims at providing a framework for assessing the detection and localization performance of guided wave-based structural health monitoring (SHM) imaging systems. The assessment exploits a damage identification metric (DIM) providing a diagnostic of the structure from an image of the scatterers generated by the system, allowing detection, localization, and size estimation of the damage. Statistical probability of detection (POD) and probability of localization (POL) curves are produced based on values of the DIM for several damage sizes and positions. Instead of relying on arduous measurements on a significant number of structures instrumented in the same way, a model-based approach is considered in this paper for estimating POD and POL curves numerically. This approach is first illustrated on a simplistic model, which allows characterizing the robustness of the SHM system for various levels of noise in test signals. An experimental test case using a more realistic case with an artificial damage is then considered for validating the approach. A good agreement between experimental and numerical values of the DIM and derived POD and POL curves is observed.
\end{abstract}

\section{Introduction}

Non-Destructive Testing (NDT) techniques are widely used in various industrial environments for characterizing structural state of pipes, tanks, shells, etc. These techniques require a human intervention for scanning meticulously the whole area, periodically or when a damage is suspected. Structural Health Monitoring (SHM) techniques can reduce maintenance costs significantly by switching from the schedule-based maintenance to a condition-based maintenance, and by allowing the use of lighter materials such as composite or sandwich panel in the case of aerospace structures [1]. SHM has been studied for decades, leading to the development of a large panel of techniques $[2,3,4,5,6,7,8,9,10,11,12,13,14,15]$. Among them, techniques based on guided wave (GW) propagation with piezoceramic transducers are cost-effective and allow a quick on-demand inspection of large areas over thin metallic and composite structures [16].

${ }^{0}(1)$ :Groupe ESEO, 10 Boulevard Jean Jeanneteau, CS 90717, 49107 Angers Cedex 2, France.

(2):GAUS - Dept. of Mechanical Engineering - Université de Sherbrooke - Sherbrooke QC, Canada, J1K 2 R1.

(3):LUNAM Université, LAUM, CNRS UMR 6613, Le Mans, France. 
Nowadays, SHM techniques are mature enough for being applied in military or in civil area. The ARP6461 guidelines provide a framework for the design, the implementation, and the assessment of SHM systems in aircraft applications [1]. In accordance with the ARP6461 guidelines, metrics need to be developed for characterizing the performance and the robustness of SHM techniques. The use of probability of detection (POD) curves for characterizing the SHM systems is recommended, which is defined as a statistical metric, widely employed in the NDT community. This requires a large number of inspection tasks for characterizing various configurations of damage that can potentially appear in the structure $[17,18,19]$. Many works have been done to extend this concept to SHM [20, 21, 22, 23, 24, 25, 26, 27, 28, 29, 30, 31, 32, 33, 34, 35, 36, 19, 37]. Recently, Janapati et al. characterized the performance of a pitch-catch SHM system for detecting a crack on an aluminum plate [19]. In this study, thirty identical coupons instrumented in the same way are considered, thus providing the statistical evolution of a damage index (DI) over several factors such as the size and the orientation of the damage, the variations of mechanical properties of the structure, and the adhesive layer below transducers. The authors conclude that duplicating coupon tests is a costly, time consuming and thus, not adapted approach for quantifying SHM systems. Nevertheless, this limitation can be overtaken by using a model-assisted approach, which produces so called ModelAssisted Probability of Detection (MAPOD) curves [21, 25, 26, 35]. This approach, more adapted to SHM, was applied for assessing performance of various GW-based SHM systems [22, 29, 36, 37]. However, SHM system assessment is generally limited to Technology Classification Level (TCL) 1 (i.e. damage detection), and only few works covering TCL 2 (i.e. damage localization) and TCL 3 (i.e. size estimation) have been performed [8, 10, 38, 27, 28, 39, 40, 41, 42, 29, 30, 43, 44].

GW-based imaging techniques provide an image of the structure which is analyzed in order to extract information about the presence of defects, their locations and their sizes. Several metrics have been developed for characterizing the ability of this class of techniques to identify the damage position and image quality, such as the exponential factor [38], which calculation is based on the noise level of the image, the amplitude of peaks, and the distance of the maximum pixel index from the actual damage position, the Localizer Operating Characteristic (LOC) curve [27], which estimates the probability of correctly localizing the damage, and the error of localization [10, 29, 42]. These metrics were used for comparing the performance of a large number of techniques. However, these studies focus on the localization assessment only, assuming that the damage was already detected. Thus, the ability of imaging techniques for detecting a damage should also be considered.

This paper aims at providing a framework for assessing the ability of GW-based SHM imaging systems to identify a damage in a structure, which includes the detection, localization and size estimation. A model-based approach is herein proposed, which consists in simulating damage identification tests with a model considering not only damage properties (type, size, position, orientation), but any disturbing parameters, such as environmental variations (temperature, humidity), operational conditions (internal stresses, loading conditions), and instrumentation considerations (noise level, sensors degradation, and debonding).

First, the general principle of the GW-based imaging SHM system assessment is presented and compared to the classical approach used in NDT. Then, the model-based approach is illustrated through the evaluation of the robustness of a simplistic SHM system. An experimental SHM system assessment is then compared to the model-based approach using a finite element model (FEM) of the set-up. The validity and limitations of the proposed model-based approach are finally discussed, 
and guidelines are formulated for producing a relevant statistical assessment.

\section{Assessment of NDT system vs. SHM system}

The performance of a monitoring system must be assessed for various damage parameters, but also environmental variations, operational conditions and instrumentation considerations [19]. The classical assessment of a NDT system and the assessment of a GW-based imaging SHM system are summarized in Fig. 1.

\subsection{Assessment of a NDT system}

The assessment of a NDT system requires the calculation of the POD curve, which is defined as the probability of detecting a damage as a function of its size $a$, and the calculation of confidence interval $[18,17]$. The $95 \%$-confidence interval is often considered, denoting the interval in which a new evaluation of the POD curve will belong $95 \%$ of the time. Therefore, this value will be considered in the present study. The gold standard metrics for characterizing the performance of a NDT system are the minimum damage size which can be detected with a probability of $90 \%$, noted $a_{90}$ and its $95 \%$-minimum confidence interval, noted $a_{90 / 95}$. The assessment employed for characterizing NDT systems, is presented in Fig. 1.a. It consists in performing detection tests on a given number $L$ of samples in a controlled environment. Tests must be repeated by a given number $B$ of operators in order to consider the non-reproducibility intrinsic to the human inspection. NDT systems are classified in two categories which determines the calculation of the POD curve [18]. Systems which give only qualitative information about the presence or the absence of damage provide hit/miss data. The second category of NDT systems provides $\hat{a}$ vs $a$ data, where $a$ is the controlling variable, which can be the damage size, length, or any parameter related to it, and $\hat{a}$ is the response of the system for such a damage. These systems give an additional quantitative information about the size of the damage. This approach is herein considered for assessing the performance of GW-based imaging systems. For calculating the POD curve, an ordinary linear regression law is determined between the controlling variable and the response of the system. Such a regression is expressed as [18]:

$$
\hat{a}=\beta_{0}+\beta_{1} a+\varepsilon .
$$

In $\operatorname{Eq}(1), \beta_{0}$ and $\beta_{1}$ are the regression parameters. Normally distributed errors $\varepsilon$ with zero mean and uniform variance are assumed (homoscedasticity), and observations must be uncorrelated. The POD curve is then obtained using the following expression [18]:

$$
\operatorname{POD}(a)=\Phi\left(\frac{a-\mu}{\sigma}\right)
$$

where $\Phi($.$) is the normal cumulative distribution function, \mu=\left(\hat{a}_{\mathrm{th}}-\beta_{0}\right) / \beta_{1}$, where $\hat{a}_{\mathrm{th}}$ is the decision threshold (i.e. the value above which the signal is interpreted as a hit), and $\sigma=\sigma_{\varepsilon} / \beta_{1}$, where $\sigma_{\varepsilon}$ is the root mean squared error of the regression. Confidence intervals are generally determined using the Wald method or the likelihood ratio criterion $[45,18]$. For a NDT system, the assessment of the localization is not necessary since such a system gives a real-time detection signal during the manual scan of the structure. Localization and detection are then intrinsically related. Moreover, assuming that the operator scans meticulously the whole area of inspection, the position 
(a) NDT system assessment

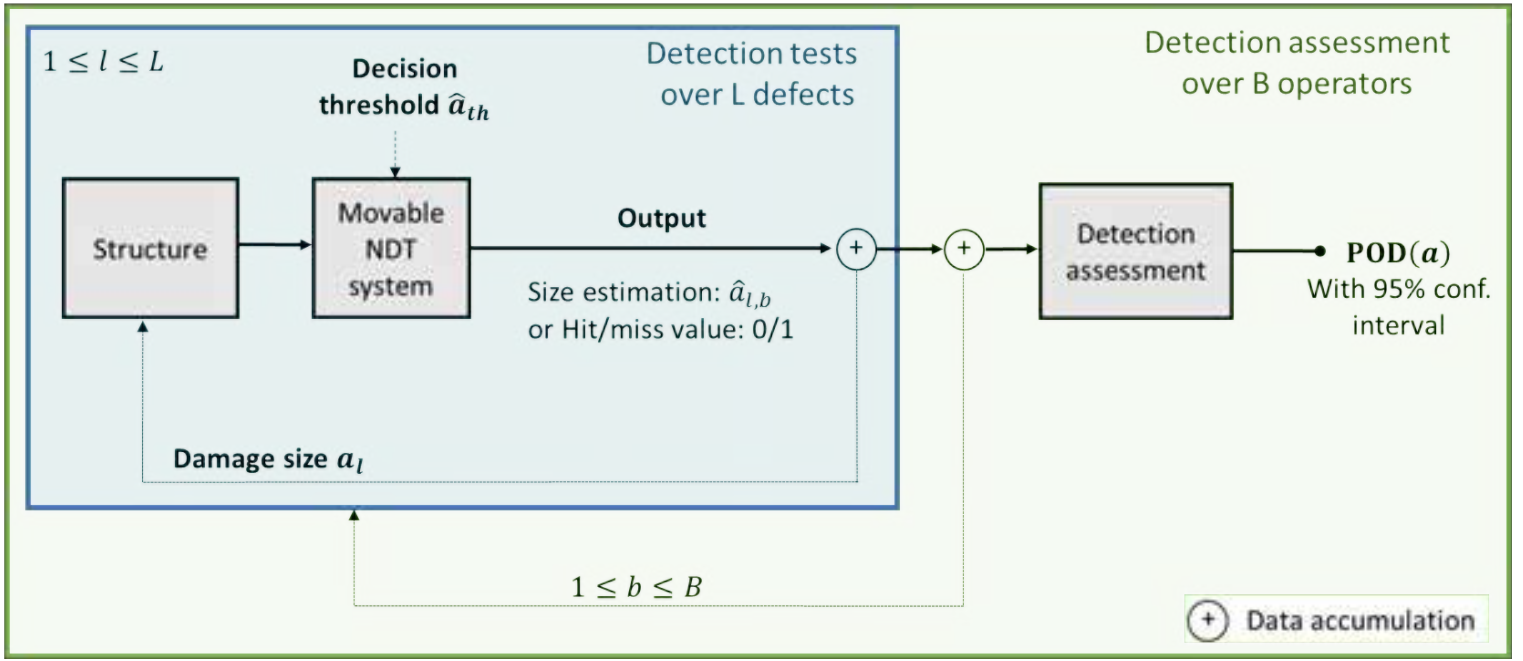

(b) SHM system assessment

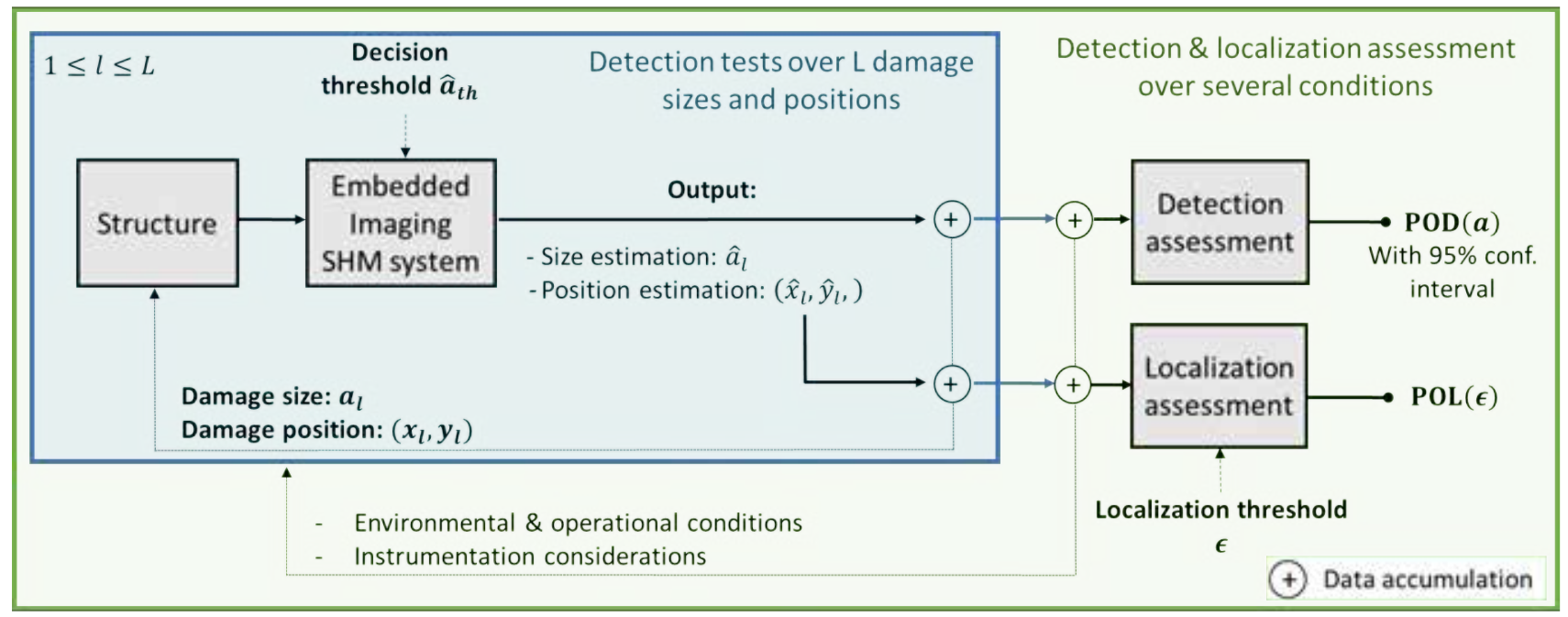

Figure 1: (a) NDT system assessment and (b) SHM system assessment.

of the damage is not a relevant parameter in the detection assessment. Also, NDT inspections are generally performed on inoperative equipment, in a controlled environment, whereas SHM systems monitor equipments in operation, and are therefore expected to operate under various disturbing conditions. 


\subsection{Assessment of a guided wave-based imaging SHM system}

Unlike NDT systems, SHM systems consider sensors permanently embedded on the structure. A GW-based imaging SHM system provides an image which reflects all the events that affect the propagation of GW in the structure. The measured effect of the acoustic interaction with the damage is thus very dependent on the SHM system configuration. For instance, if a damage appears far from the transducers, it will be less detectable since it will have a weak effect on the acoustic signal. Therefore, the damage position relative to the transducers positions is a relevant parameter, and the assessment of a SHM system must cover the whole possible damage positions. For establishing the diagnostic of the structure, a spatial damage index (DI) is first computed over the image of the whole structure, then a global damage identification metric (DIM) $\hat{a}$ is extracted from this image, giving information about the presence of a damage, its position, and size. It has been shown that the maximum value of the imaging DI monotonously increases with the damage size [8] and has a Gaussian distribution over the damage position [40], making it a suitable candidate for performing a POD analysis. Therefore, the DIM is defined as the maximum pixel value of the DI, and the classical $\hat{a}$ vs. a POD formulation is herein considered for assessing detection performance of the system, allowing the derivation of $a_{90}$ and $a_{90 / 95}$ values as in section Assessment of a NDT system.

Several metrics have been proposed for estimating the probability of localizing a damage such as the LOC curves[27] and the cumulative probabilistic distribution function with respect to the localization error[42]. The approach proposed in the present study is inspired from both of them. It consists in evaluating the probability of the damage to be localized within a tolerance $\epsilon$ expressed as an absolute distance with the actual damage localization [44]. The absolute error of localization (AEL), which is the Euclidean distance between the real damage position $\left(x_{a}, y_{a}\right)$ and its estimation $\left(\hat{x}_{a}, \hat{y}_{a}\right)$, is expressed as:

$$
A E L=\sqrt{\left(\hat{x}_{a}-x_{a}\right)^{2}+\left(\hat{y}_{a}-y_{a}\right)^{2}} .
$$

The localization is assumed to be correct if the estimated position falls into a circle of center $\left(x_{a}, y_{a}\right)$ and radius $\epsilon$. An estimation of the probability of localization (POL) is thus naturally given by the the ratio of correct localization, which is expressed as a function of $\epsilon$ :

$$
\operatorname{POL}(\epsilon)=\frac{1}{K} \sum_{j=1}^{K} H\left(\epsilon-A E L_{j}\right) .
$$

In Eq.(4), $H($.$) is the Heaviside-step function, and K$ refers to the total number of localization tests. Therefore, the performance of the localization is evaluated with the minimum AEL value for which $90 \%$ of defects are correctly localized, and is noted $\epsilon_{90}$. Contrary to the POD formulation, which is derived from a linear regression over a large number of DIM, the POL curve is given directly by the ratio of defects correctly localized. Therefore, the confidence interval has no signification in this case.

\section{Guided wave-based SHM system assessment}

\subsection{GW imaging configuration}

To illustrate the proposed approach, a simplified embedded SHM imaging system, composed of three piezoelectric transducers bonded on an infinite aluminum plate is considered, such as 


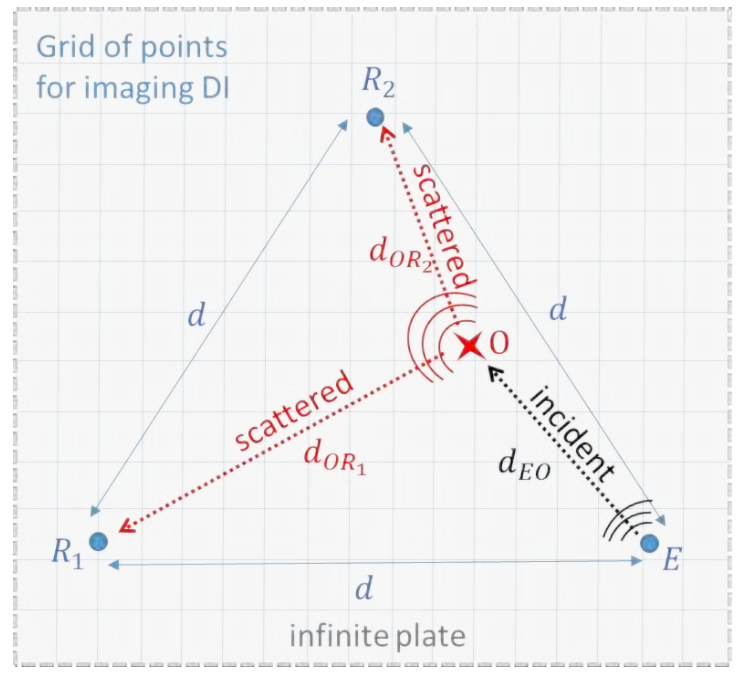

Figure 2: Illustration of the simplified SHM system considered.

represented in Fig. 2. The transducers are circular with a radius $r$, and the array forms an equilateral triangle of side length $d$ for simplicity. To perform an image, one transducer, noted $\mathrm{E}$ in Fig. 2, emits an ultrasound burst which propagates through the structure and which is recorded by the two other transducers, noted $R_{1}$ and $R_{2}$. Each transducer acts as an emitter in turn whilst the others are receivers (round-robin procedure) such that 3 signals, corresponding to the 3 possible emitter-receiver pairs are acquired. The signals relative to each couple $n$ of emitter-receiver when the structure is pristine are called baseline and are noted $\mathbf{s}_{n}^{\text {baseline }}$. This procedure is repeated for performing a detection test when a damage is present and located at the position $\mathrm{O}$ in Fig. 2. Test signals $\mathbf{s}_{n}^{\text {test }}$ are then recorded and the residual signals $\mathbf{r}_{n}=\mathbf{s}_{n}^{\text {test }}-\mathbf{s}_{n}^{\text {baseline }}$ relative to the $n$th pair of emitter-receiver are introduced into an imaging algorithm that tests the possible positions of the damage on the structure. An image of the scatterers is obtained by calculating a DI associated to each position tested, noted $\mathrm{I}(x, y)$, using the information of residual signals relative to each pair of emitter-receiver. The image illustrates the variations that have affected the propagation of GW in the structure, with respect to the baseline acquisition. Various damage imaging algorithms exist such as Delay-And-Sum (DAS) [8], also referred as Embedded Ultrasonic Structural Radar (EUSR) [7], Correlation-Based (CB) also referred as Excitelet [9], Block-Sparse Basis Pursuit Denoising (BPDN) [46], Generalized Cross-Correlation with Phase Transform (GCC-PHAT) [47]. In order provide statistically representative metrics, a large amount of detection tests must be realized numerically, which may represent a huge computational time. The gold standard DAS method is the one that requires the least mathematical operation while offering satisfying results [44] and will be considered in this paper. This algorithm assumes that the acoustic signal propagates through the material without any dispersion, which is a correct approximation only for non dispersive GW modes. For overtaking this limitation, a pre-processing is applied to residual signals that compensates the dispersion effect [48] but does not consider the multiple mode propagation and 
the mode conversion. The following expression gives the DI obtained with the DAS algorithm:

$$
\mathrm{I}(x, y)=\frac{1}{N} \sum_{n=1}^{N} \int_{-\infty}^{+\infty} \sqrt{d_{\mathrm{E}_{n}} d_{\mathrm{R}_{n}}}\left|\mathcal{H}\left(\mathbf{r}_{n}^{c}\right)\right| \delta\left(t-\left[\frac{d_{\mathrm{E}_{n}}}{c_{g}}+\frac{d_{\mathrm{R}_{n}}}{c_{g}}+t_{b}\right]\right) d t .
$$

In Eq.(5), $\mathbf{r}_{n}^{c}$ is the residual signal with compensation of dispersion [48], $\delta($.$) is the Dirac delta$ function, and $N$ refers to the number of couples of emitter-receiver. The envelop of the compensated residual signal is considered by taking the absolute value of its Hilbert transform, noted $\mathcal{H}($.$) . The geometrical attenuation is compensated by multiplying the contribution of each pair of$ emitter-receiver by $\sqrt{d_{\mathrm{E}_{n}} d_{\mathrm{R}_{n}}}$, where $d_{\mathrm{E}_{n}}$ and $d_{\mathrm{R}_{n}}$ are the distances between the emitter $\mathrm{E}_{n}$ and the considered pixel at the coordinates $(x, y)$, and between the pixel and the receiver $\mathrm{R}_{n}$, respectively. $c_{g}$ is the group velocity of the considered mode at the inspection frequency. Subtracting the half duration of the input signal $t_{b}$ eliminates the bias in the evaluation of the total distance traveled by the wave.

\subsection{Introduction to the model-based assessment}

As reported in the literature, an experimental assessment of the SHM system would require a large amount of coupons instrumented in a similar way. A model-based approach is however herein considered, which consist in performing numerical detection tests for producing MAPOD and MAPOL curves. This section illustrates the principle of the model-based SHM system assessment. The pin-force model is used to model GW generation by the transducers, considering the simple configuration of the SHM system for the purpose of validation $[49,50]$. To model the acoustic propagation, a far-field approximation is also assumed. Only the propagation of the $A_{0}$ mode is considered, and the plate edge reflections are neglected for simplicity. The damage is assumed to be equivalent to an omnidirectional point-like reflector which amplitude is assumed to grow as the size of the damage increases. A more complex representation of the damage would implementing the directivity pattern of the damage $[51,52,53]$.

Taking into account all these considerations, the direct transfer function $T_{\mathrm{ER}}$ between an emitter $\mathrm{E}$ and a receiver $\mathrm{R}$ is expressed in the frequency domain by [49]:

$$
T_{\mathrm{ER}, n}(\omega) \propto H_{0}^{(2)}\left(k_{A_{0}} d_{n}\right),
$$

where $d_{n}$ is the distance between the emitter and the receiver of the $n$th pair, $\omega$ is the angular frequency, $H_{0}^{(2)}$ is the Hankel function of second kind and zero order, and $k_{A_{0}}$ denotes the wavenumber associated to the considered propagating mode $A_{0}$. When a damage is located at a point $\mathrm{O}$ with a scattering coefficient of $\zeta$ on the plate, the theoretical transfer function $T_{\mathrm{EOR}}$ between an emitter $\mathrm{E}$ and a receiver $\mathrm{R}$ after scattering by the damage in $\mathrm{O}$ is defined by [54]:

$$
T_{\mathrm{EOR}, n}(\omega) \propto \zeta H_{0}^{(2)}\left(k_{A_{0}} d_{\mathrm{EO}, n}\right) H_{0}^{(2)}\left(k_{A_{0}} d_{\mathrm{OR}, n}\right) .
$$

In Eq.(7), $d_{\mathrm{EO}, n}$ and $d_{\mathrm{OR}, n}$ are the distances between the emitter and the damage, and between the damage and the receiver, respectively, as presented in Fig. 2. Baseline signals are calculated assuming that the structure is pristine. Then, the direct signal at the receiver corresponding to the $n$th pair of transducers is calculated using Eq.(6):

$$
s_{n}^{\text {direct }}(t)=E(t) * \mathcal{F}^{-1}\left[T_{\mathrm{ER}, n}(\omega)\right],
$$


where $(*)$ represents the convolution operator, $E(t)$ is the excitation signal, and $\mathcal{F}^{-1}$ refers to the inverse Fourier transform of $T_{\mathrm{ER}, n}$. Baseline signals are finally expressed as:

$$
s_{n}^{\text {baseline }}(t)=s_{n}^{\text {direct }}(t)+b_{n}^{\text {baseline }}(t) .
$$

In Eq.(9), $b_{n}^{\text {baseline }}(t)$ is a random white Gaussian noise which is added to the direct signal for simulating an instrumentation noise. However, baseline signals are assumed to be recorded in a favorable controlled environment, such that a Signal-to-Noise Ratio (SNR) of $50 \mathrm{~dB}$ is always considered in baseline signals. Similarly, the time domain signal scattered by the damage is calculated using Eq.(7):

$$
s_{n}^{\text {scat }}(t)=E(t) * \mathcal{F}^{-1}\left[T_{\mathrm{EOR}, n}(\omega)\right] .
$$

When a damage is present in the structure, the signal acquired by the receiver is the sum of the two contributions given by Eq.(8) and Eq.(10). The signal relative to the $n$th pair of emitter-receiver when a damage is present is then given by:

$$
s_{n}^{\text {test }}(t)=s_{n}^{\text {scat }}(t)+s_{n}^{\text {direct }}(t)+b_{n}^{\text {test }}(t) .
$$

In Eq.(11), $b_{n}^{\text {test }}(t)$ is a random white Gaussian noise. In this case, the SNR is a priori unknown since it depends on the conditions of inspection of the structure. If the test signal is not affected by environmental or operational variations, the residual signal $\mathbf{r}_{\mathbf{n}}$ is obtained by subtracting Eq.(11) from Eq.(9):

$$
r_{n}(t)=s_{n}^{\text {scat }}(t)+b_{n}^{\text {residual }}(t),
$$

where $b_{n}^{\text {residual }}(t)=b_{n}^{\text {test }}(t)-b_{n}^{\text {baseline }}(t)$ has the distribution of a white Gaussian noise.

\subsection{Detection assessment}

The imaging process is performed over a $400 \mathrm{~mm}$ side-square image with a resolution of $2 \mathrm{~mm}$, centered in the middle of the plate. For establishing the diagnostic of the structure, the $\hat{a}$ vs. $a$ approach is considered. The size of the damage is parametrized by the scattering index $\zeta$ in Eq.(7) assuming an omnidirectional scattering pattern. The DIM, which is the maximum of the DI over the whole imaging area, gives directly the estimation of the scattering index, and is therefore noted $\hat{\zeta}$. The estimation of the damage position is given by the coordinates $\left(\hat{x}_{\zeta}, \hat{y}_{\zeta}\right)$ of the DIM.

A Monte-Carlo procedure is considered for performing 1000 detection tests, assuming random damage sizes and positions over the plate. For illustrating the robustness of the SHM system, SNR of $20 \mathrm{~dB}$ and $50 \mathrm{~dB}$ are considered for the test signal in Eq.(11). Values of the DIM $\hat{\zeta}$ obtained for the various damage configurations, and SNR are represented in Fig. 3.a as a function of the scattering index $\zeta$. When the SNR is equal to $50 \mathrm{~dB}$, the noise barely affects the signals, and a linear dependency is observed between the DIM $\hat{\zeta}$ and the scattering index $\zeta$ when $\zeta>\zeta_{\min }^{50 \mathrm{~dB}}=0.02$, which correspond to the minimum identifiable scattering index. Therefore, we set the decision threshold $\hat{\zeta}_{t h}^{50 \mathrm{~dB}}$ just above the higher value of $\hat{\zeta}$ in the range $\left[0, \zeta_{m i n}^{50 \mathrm{~dB}}\right]$. In this case, the decision threshold $\hat{\zeta}_{t h}^{50} \mathrm{~dB}=0.02$. When the SNR is equal to $20 \mathrm{~dB}$, the noise amplitude is increased, leading to imaging artifacts. For scattering indices $\zeta<0.3$, these artifacts are higher than the amplitude scattered by the damage, which is therefore not visible in the image produced, as one can see in Fig. 3.b. This is manifested by an offset of the DIM in Fig. 3.a, which value is equal to the noise amplitude. The minimum identifiable scattering index $\zeta_{\min }^{20 d B}$ is therefore equal to 0.3 . The decision threshold is then increased to $\hat{\zeta}_{t h}^{20} \mathrm{~dB}=0.42$. In both cases, the DIM shows a Gaussian distribution 


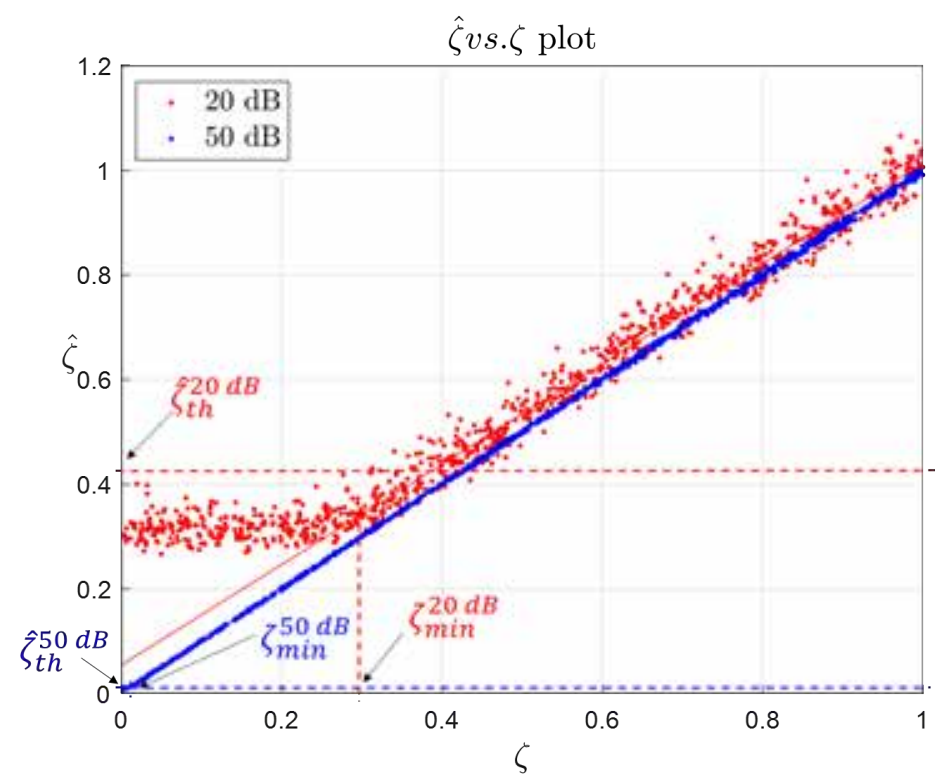

(a)
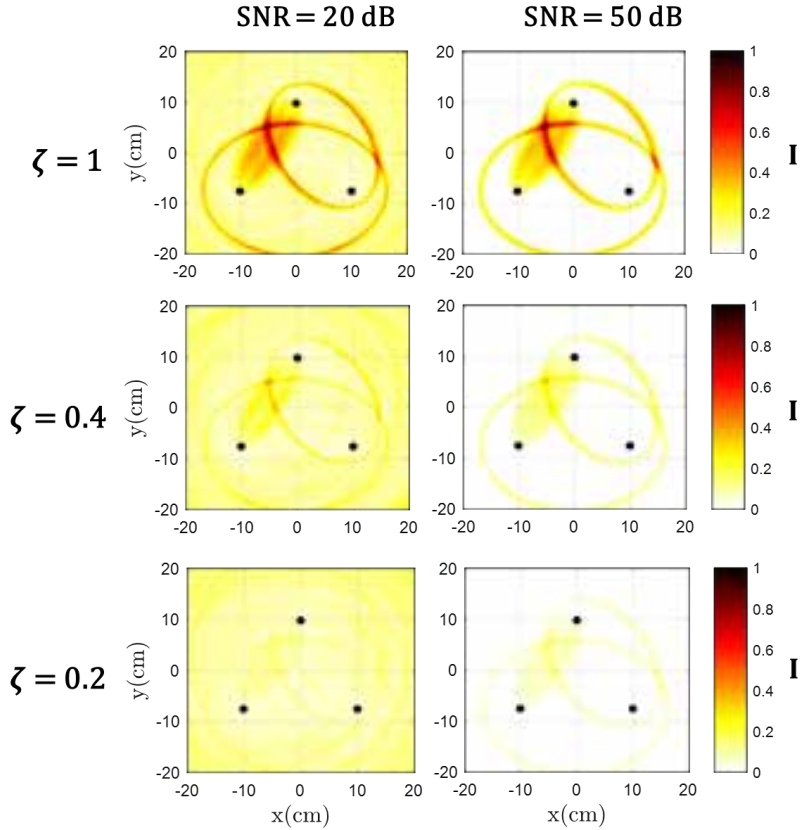

(b)

Figure 3: (a) Values of DIM $\hat{\zeta}$ obtained for 1000 random scattering indices $\zeta$ and positions, as a function of the scattering index $\zeta$, assuming a white Gaussian noise added to residual signals with a SNR equal to $50 \mathrm{~dB}$ (blue) and $20 \mathrm{~dB}$ (red); lines: regression calculated over the linear region $\zeta \geq \zeta_{\min }$. (b) Typical images obtained with the DAS-DC algorithm when a damage is located at the position $(-5 \mathrm{~cm}, 5 \mathrm{~cm})$, for which scattering index $\zeta$ is equal to $0.2,0.4$ and 1 , and for residual signals with SNR equal to $20 \mathrm{~dB}$ and $50 \mathrm{~dB}$; black dots: piezoelectric transducers.

with constant variance. Therefore, statistical conditions required for producing a POD curve based on a $\hat{a}$ vs. $a$ approach are satisfied in the range of scattering indices $\zeta>\zeta_{\min }$. Therefore, linear regressions are calculated over the linear domain $\zeta>\zeta_{\min }$ in both cases using the maximum likelihood estimation and are represented in Fig. 3.a. The confidence intervals give a relevant information when a regression is performed over a limited number of sample. In the present case, 1000 points are considered, such that a high statistical confidence can be attributed to the regression curve. Therefore, confidence intervals are not represented in Fig. 3.a.

POD curves calculated using the $\hat{a}$ vs. $a$ formulation described by Eq.(2), and corresponding to SNR equal to $50 \mathrm{~dB}$ and $20 \mathrm{~dB}$ are represented in Fig. 4, considering a decision threshold equal to $\hat{\zeta}_{t h}^{50 \mathrm{~dB}}$ and $\hat{\zeta}_{t h}^{20 \mathrm{~dB}}$, respectively. When the SNR is equal to $50 \mathrm{~dB}$, the POD curve is equal to 0 until $\zeta \approx 0.02$, where it increases sharply to $100 \%$. This sharp increase is due to the small variance of the data. By definition, the decision threshold is the $\hat{\zeta}$ value associated with $50 \%$ POD [18]. The minimal identifiable scattering index detectable with a probability of $90 \%$, noted $\zeta_{90}^{50} \mathrm{~dB}$ in Fig. 4 , 


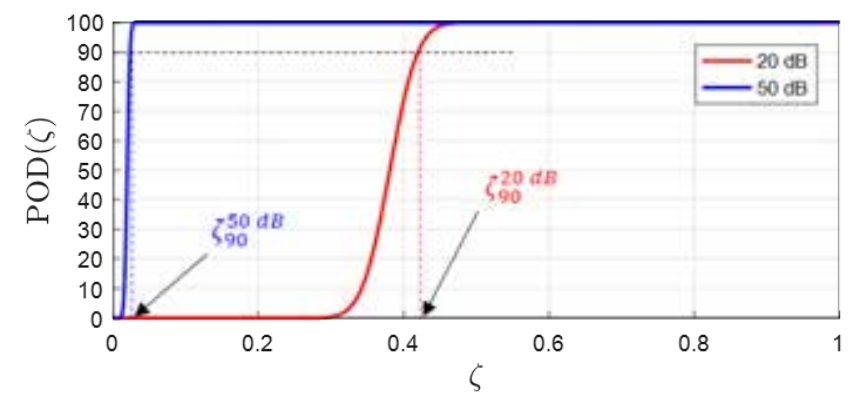

Figure 4: POD curves obtained with the â vs. a formulation, assuming a SNR of $50 \mathrm{~dB}$ (blue) and $20 \mathrm{~dB}$ (red).

is therefore approximately equal to the decision threshold $\hat{\zeta}_{t h}^{50 \mathrm{~dB}}$. When the SNR is equal to 20 $\mathrm{dB}$, the POD is equal to $0 \%$ for $\zeta<0.3$ and increases progressively to reach $100 \%$ for $\zeta \geq 0.48$. In this case, the variance of DIM is increased compared to the case at $20 \mathrm{~dB}$, which explains the slower increases of the POD curve. value $\zeta_{90}^{20} \mathrm{~dB}=0.42$ is obtained.

\subsection{Localization assessment}

POL curves based on detected damages are illustrated in Fig. 5 as a function of the localization threshold $\epsilon$. When the SNR is equal to $50 \mathrm{~dB}$, damage positions are perfectly identifiable, as one

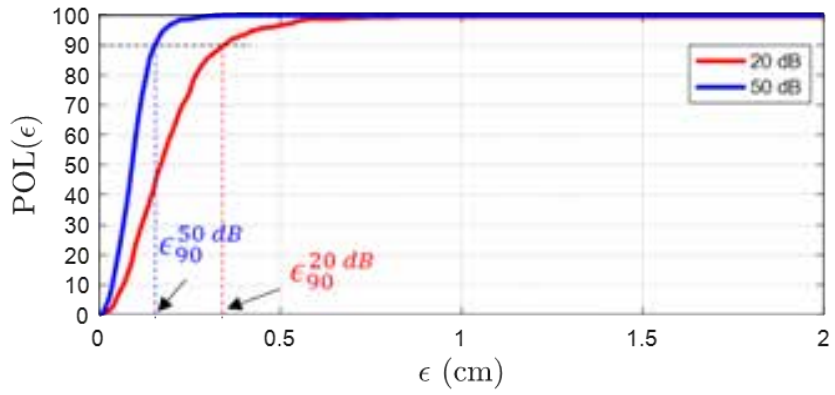

Figure 5: POL curves calculated over the detected damages, assuming a SNR of $50 \mathrm{~dB}$ (blue) and $20 \mathrm{~dB}($ red).

can see in Fig. 3.b. Therefore, $90 \%$ of the detected damages are located within an AEL of 0.15 $\mathrm{cm}$. When the SNR is equal to $20 \mathrm{~dB}, 90 \%$ of the damages detected are localized within an AEL of $0.35 \mathrm{~cm}$. The localization performance is then barely impacted by the noise such that acceptable localization performance are obtained at $20 \mathrm{~dB}$. Obviously, reducing the decision threshold would lead to a higher number of non identifiable damages and would decrease the POL curve at 20 dB. Selecting an appropriate decision threshold is then crucial for ensuring acceptable localization performance. 


\section{Application of the model-based assessment approach}

\subsection{Experimental set-up}

In order to provide an experimental comparison basis for the proposed assessment approach, a SHM system is considered, which configuration is presented in Fig. 6. Three circular piezoelectric
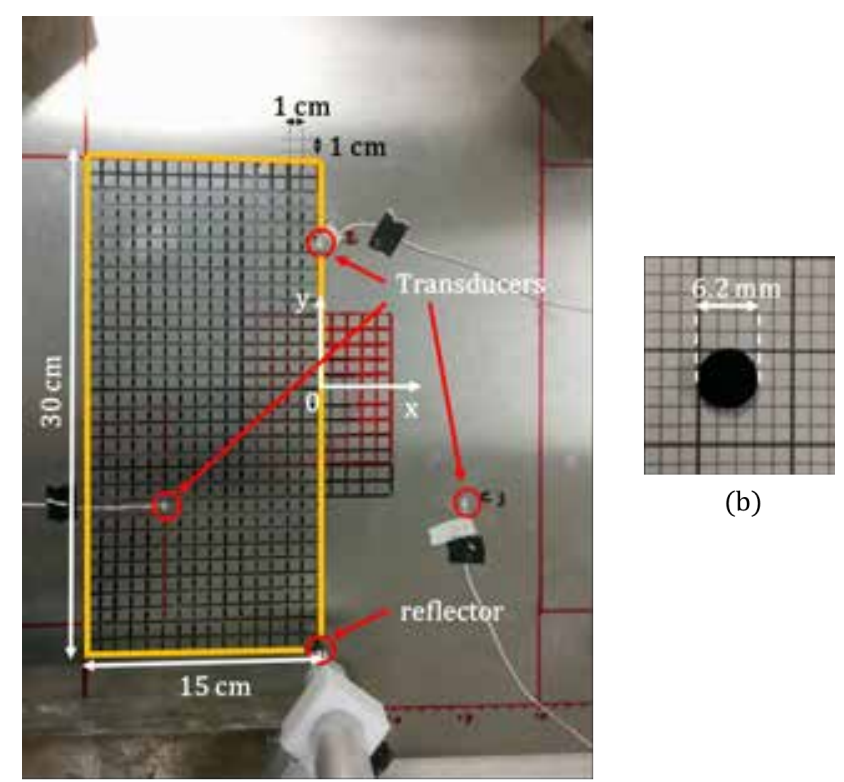

(b)

(a)

Figure 6: (a) Experimental set-up, and (b) example of a viscoelastic disc of diameter equal to 6.2 $\mathrm{mm}$, simulating an omnidirectional reflector.

transducers PZT-5A of $5 \mathrm{~mm}$ diameter and $0.5 \mathrm{~mm}$ thickness are bonded with epoxy on a 6061-T6 aluminum plate. An absorbing paste is used for minimizing the acoustic reflections by the edges of the plate, thus simulating as much as possible an infinite medium. The damage is simulated with a viscoelastic disc, which is represented in Fig. 6.b, loaded with a mass of $500 \mathrm{~g}$ on the aluminum plate. Such a simulated damage was suggested for simulating an omnidirectional reflector [26]. Such a system is quite simple, and allows to perform repeatable measurements. A HP 33120A function generator and a Novo Electronics UA8200 amplifier are used for generating a 1.5-cycles burst at $100 \mathrm{kHz}$, which was evaluated as a good compromise between the time length and the frequency spreading. A National Instrument NI-5105 acquisition device with a custom LabVIEW interface is used for recording the response voltage signal generated by the two other transducers and for synchronizing the generation and the acquisition. For each detection tests, the signals averaged over 300 acquisitions are considered, which reduce the measurement noise, leading to reproducible detection tests. For assessing the performance of this SHM system as a function the damage diameter $a, 8$ simulated damage diameters of $2.1,2.6,3.2,3.7,4.0,4.3,4.7$, and $6.2 \mathrm{~mm}$ are considered. For each simulated damage diameter, detection tests are performed over 100 positions chosen arbitrary over the black grid in Fig. 6.a. All the detection tests are performed on the same 
structure, with a single SHM system bonded on it, which can raise a question about the statistical independence of observations. Therefore, a baseline signal is acquired prior to each detection test. As in section Introduction to the model-based assessment, experimental or environmental conditions are not varied between the baseline acquisition and the detection test, and each residual signal can be considered as independent. Images are then produced using the DAS-DC algorithm as in section Introduction to the model-based assessment.

\subsection{Numerical set-up}

In section Introduction to the model-based assessment, the approach was illustrated using the pin-force model, assuming an artificial omnidirectional point-like reflector. Such a model allows assessing the method and limitations of the approach, but the geometry of the realistic damage must be considered for assessing the performance of the SHM system as a function of the damage size on a given configuration. In order to demonstrate the model-based approach, a FEM of the the experimental SHM system performed using COMSOL Multiphysics 5.2 is considered. The damage is modeled by a polymer disc on the plate, with a thin elastic layer between the disc and the plate which has a thickness $h_{a}=0.05 \mathrm{~mm}$, and given normal and tangential spring constants. Transfer functions between the input signal and the measured voltage at the other transducers are calculated, for frequencies varying from $20 \mathrm{kHz}$ to $200 \mathrm{kHz}$ with a step of $3 \mathrm{kHz}$, which appears to be sufficient. As a calibration of our FEM, the normal and tangential spring constants are adjusted in order to get a good match between numerical and experimental transfer functions in both transmission and in reflection. Cubic elements of maximum size equal to $\lambda_{A_{0}} / 5$ are considered for meshing the plate, where $\lambda_{A_{0}}$ is the wavelength associated to the mode $A_{0}$ at the frequency of interest. Absorbing layers are used in order to prevent edge reflections. Therefore only the mode $A_{0}$ is considered in the plate. Tetrahedral elements of maximum size $h_{p z t} / 2$ are considered for modeling the transducers and the damage, where $h_{p z t}$ is the thickness of transducers. A Monte-Carlo procedure is then performed for simulating residual signals corresponding to 168 random polymer disc diameters between 0.1 and $9 \mathrm{~mm}$, and positions over the $400 \times 400 \mathrm{~mm}$ plate, and images are produced using the DAS-DC algorithm.

\subsection{Detection and localization assessment}

In order to obtain homoscedastic data with a Gaussian error, a Box-Cox transformation with a transformation factor equal to 0.1 is applied to the DIM values [55]. Those transformed DIM correspond to the $\hat{a}$ values, whereas $a$ is the simulated damage diameter in centimeters. A linear relationship is obtained by taking the logarithm of $\hat{a}$ and of $a$ values. Other regularization process are possible such as Piecewise model or nonparametric POD estimation [56, 57]. In Fig. 7, the $\log (\hat{a})$ vs. $\log a$ data obtained (a) numerically and (b) experimentally are represented. Linear regression curve calculated over the data are also represented in both cases in Fig. 7 . In the numerical case, the linear regression is calculated in the region where results are homoscedastic, until $\log (a)=-0.7$. The best linear fit is obtained with a coefficient of determination $R^{2}$ equal to 0.96 in the numerical case and 0.75 in the experimental case. The 95\%-confidence intervals associated to the linear regression calculated with the likelihood ratio criterion are also represented in Fig. 7. A high dispersion of experimental data is observed compared to numerical data, which can be attributed to our experimental procedure (misplacement of the simulated damage for different repetitions of the measurement). This leads to a variation of the damage identification metric. 
(a)
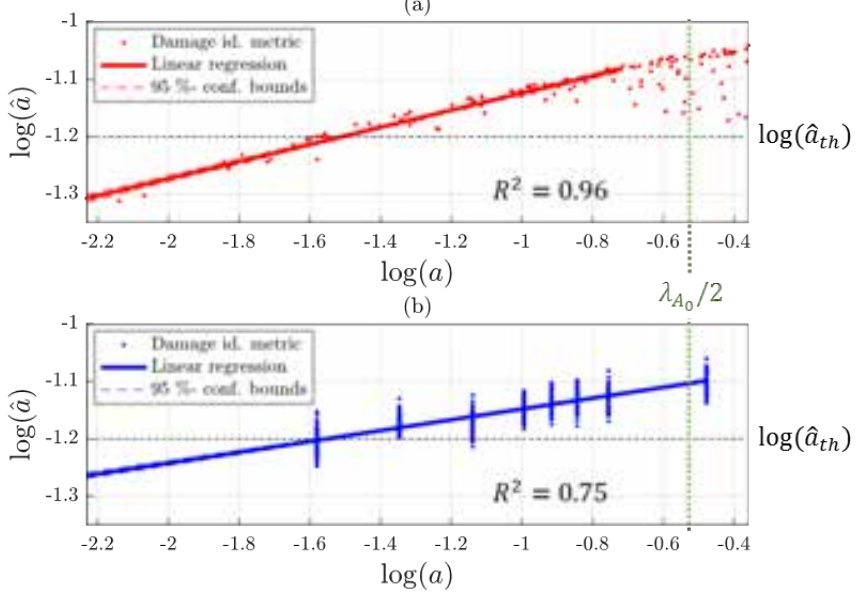

Figure 7: Log-log representation of the damage identification metric as a function of the simulated damage diameter (dots), and linear regressions (solid lines) with their respective $95 \%$-confidence intervals (dotted lines). In (a), red color refers to numerical detection tests performed over 168 random simulated damage positions and diameters, whereas in (b) blue color refers to experimental detection tests, performed over 8 simulated damage diameters and 100 random positions per diameter.

Moreover, the slight difference observed between the regression curves obtained numerically and experimentally can be attributed to the contact between the viscoelastic disc and the aluminum plate which is imperfectly modeled with FEM. Despite this result, the experimental and the numerical linear regressions are very close, which validate the model-based approach of the inspection task.

The linear regression must be done on the homoscedastic data for the POD curve to be properly calculated. In the numerical results, data are homoscedastic until $\log (a)=-0.7$ and becomes heteroscedastic above this value. When the size of the simulated damage is smaller than the half wavelength of the mode considered, here $\lambda_{A_{0}} / 2$, the scattering pattern of the simulated damage can be assumed omnidirectional (i.e. the scattered amplitude is independent of the position of the simulated damage). When the size of the simulated damage approaches $\lambda_{A_{0}} / 2$, the scattered amplitude (and so the DIM) depends on the position of the simulated damage. In experimental data, this effect is masked by the high dispersion due to the misplacement of the simulated damage as explained previously, such that data appears homoscedastic whatever the size of the simulated damage.

The confidence intervals of the experimental and numerical linear regressions in Fig. 7 are both very tights. In the numerical results, this is explained by the high $R^{2}$ value which compensates the small amount of points considered for calculating the regression. Experimentally, the low $R^{2}$ value is compensated by the high amount of data considered for the regression calculation. It is important to note that the regression curve obtained experimentally is not included into the confidence intervals obtained numerically because the error due to the misplacement effect is not taken into account in our numerical model. 

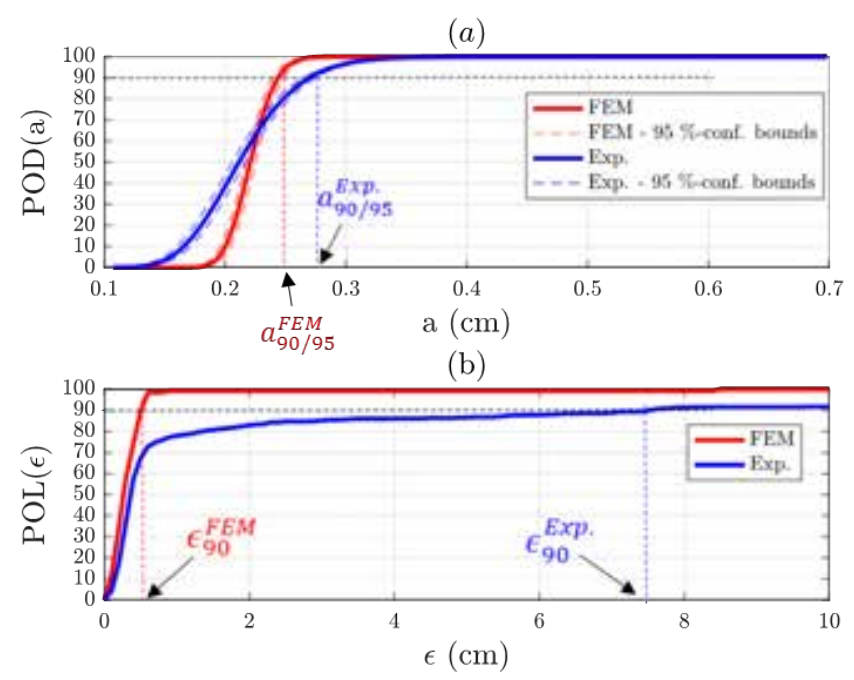

Figure 8: (a) POD curves obtained experimentally (solid blue line) and with the FEM (solid red line), and their respective $95 \%$-confidence interval (dotted lines); (b) POL curves obtained experimentally (blue) and with the FEM (red). The decision threshold $\log \left(\hat{a}_{\mathrm{th}}\right)=-1.2$.

In order to illustrate the POD curves, an arbitrary decision threshold $\log \left(\hat{a}_{\mathrm{th}}\right)=-1.2$ is chosen, which is represented in Fig. 7. The corresponding experimental and numerical POD and POL curves are presented in Fig. 8.a and 8.b, respectively. A good agreement is observed between the experimental and the numerical POD curves: numerically, a value $a_{90 / 95}^{\mathrm{FEM}}=0.25 \mathrm{~mm}$ is obtained whereas $a_{90 / 95}^{\text {Exp. }}=0.28$ is obtained experimentally. In Fig. 8.b, the numerical POL estimates that $90 \%$ of the simulated defects are localized within an AEL $\leq 0.5 \mathrm{~cm}$, whereas this value increases to $7.5 \mathrm{~cm}$ experimentally. This can be attributed to the fact that in some cases the three ellipses used for damage imaging do not cross exactly at the same location, and the maximum DI may correspond to another position, where two ellipses cross. Increasing the number of transducers would increase the performance of the localization by the SHM system. Also, the results could be improved by calculating the DI only in a zone around the true damage location, but this assumes that such a critical zone is identified in advance, where damages are more likely to occur.

\section{Discussion}

\subsection{SHM system assessment and design}

The objective of this paper is to illustrate the approach proposed for assessing GW-based imaging SHM techniques and the model-based assessment. Therefore a basic DIM is considered for analyzing the imaging DI produced by the SHM system, and for detecting and localizing the damage. The performance of the SHM system depends strongly on the DIM. Also, the SHM system considered for illustrating the assessment is simplistic, and only 3 transducers are considered, which is the minimum required for performing imaging. Therefore, the SHM system can be optimized in order to increase its performance. 
In this paper a model is used to simulate numerically the POD curve, which is compared to the one obtained experimentally in the same conditions which is different than a model-assisted POD. In the second case, the model is used for enriching the database. Of course, the approach presented in this paper can be used in this way. However, in SHM, acquiring experimental data in real condition is sometimes difficult and a model-based approach could be of interest.

In section Application of the model-based assessment, the variance of experimental and numerical values of the DIM increases with the damage diameter $a$ when $a \geq \lambda_{A_{0}} / 2$ (heteroscedasticity), which goes against one of the requirements for performing an ordinary linear regression. Therefore, for assessing the detection performance of a GW-based imaging SHM system, POD curve should be ideally calculated over damage sizes below $\lambda / 2$, where $\lambda$ is the wavelength of the mode considered, in order to avoid taking into account the complex directivity patterns of the damage. This observation stands in contradiction with the general requirement for optimizing the damage detection, which suggest that the damage size should be superior to $\lambda / 2$.

The optimization-based SHM design consists in finding the optimal parameters in order to reach specified performance metrics $a_{90}, a_{90 / 95}$ and $\epsilon_{90}$. Optimization can be done on the input signal (type, frequency, number of cycles) for adapting the wavelength to the expected damage type and size, on the instrumentation (type and number of transducers) for increasing the quality of images, or on the pre-processing of residual signals for compensating variations of temperature for example, and on post-processing image. Since MAPOD appears as feasible in the case of classical SHM configurations, those design optimization might be realized numerically using a representative FEM.

\subsection{Guidelines for the model-based detection and localization assessment}

Based on the method presented in this paper, guidelines are formulated for performing a relevant assessment of GW-based imaging SHM systems:

1. The assessment of the SHM system based on imaging should be based on POD and POL curves, such as the one described in the present paper.

2. If a $\hat{a}$ vs. $a$ approach is considered for calculating the POD curve, the linear regression should be calculated over homoscedastic data, which implies detection tests performed with damage sizes inferior to the half wavelength of the mode considered for imaging the DI if an omnidirectional scattering pattern of the damage is assumed. In this case, the POD curve is valid only for damage sizes inferior to this half wavelength.

3. The representativeness of the POD and the POL curves depends on the extent of data available. Therefore, the number of case test must be as large as possible. These values should correspond to different damage sizes and positions.

4. The performance of the SHM system depends on the quality of the image produced. Therefore, the image resolution must be smaller than the minimal damage size covered by the assessment, and its extent must be larger than the most remote damage location.

5. The assessment is only valid with types and positions of damage and for disturbing parameters considered in the assessment. Therefore, the assessment should cover as much realistic 
disturbing parameters as possible, including their representative range of variation. All cases not covered are beyond the scope of the assessment. Extrapolation of data should be avoided or clearly justified.

6. The assessment of the system requires a valid DIM for selecting the useful information contained in the DI imaging in order to optimize the damage detection and localization.

7. A model-based or a model-assisted assessment is recommended for performing virtual detection tests. The model is used to simulate transfer functions between each couple of emitterreceiver, first, without damage for producing baseline signals, then, when a damage is present for producing test signals. In such a case, acoustic dispersion and attenuation must be considered. Modeling the interaction with the damage requires the diffraction directivity pattern, which can be obtained numerically or experimentally. If possible, the complete acquisition chain should be modeled since it may introduce gain or distortion in signals.

8. Model simplifications must be justified by numerical or experimental results, or by state-ofthe-art references.

\section{Conclusion}

In this paper, a general procedure is introduced for assessing the performance of GW-based SHM imaging methods for detecting and localizing a damage on an infinite flat plate under ideal laboratory conditions. In future works, this approach will be expanded to a more complex mechanical structure such as composite panel or more complex geometrical structures. The detection assessment is based on $\hat{a}$ vs. a POD curves, well established in the NDT community, whereas the localization assessment is based on POL curves. A model-based procedure is considered for performing detection tests numerically instead of repetitive measurements. This procedure is first illustrated on a simplistic model, assuming a damage acting like a point-like omnidirectional reflector, which intensity is parametrized by its scattering index. The noise robustness of such a system is illustrated through POD and POL curves. For validating the model-based approach, the assessment of a real SHM system is performed experimentally and compared with a numerical assessment performed on a FEM of the system. Values of the damage identification metric against the size of the damage obtained experimentally and numerically are in accordance globally. Optimization of the SHM system can be done through the model-based assessment in order to reach specified requirements in terms of detection and localization performance. This aspect, which is beyond the scope of the present paper, will be illustrated in future work.

\section{Acknowledgment}

This study has been funded by Le Mans Acoustique (LMAc) institute and the Natural Sciences and Engineering Research Council of Canada (NSERC). The authors would like to thank Charles Annis for his help in the calculation of POD curves. 


\section{References}

[1] Guidelines for Implementation of Structural Health Monitoring on Fixed Wing Aircraft ARP6461. Technical report, SAE International, 2013.

[2] Wang CH, Rose JT and Chang FK. A synthetic time-reversal imaging method for structural health monitoring. Smart Mater Struct 2004; 13(2): 415.

[3] Ihn JB and Chang FK. Detection and monitoring of hidden fatigue crack growth using a built-in piezoelectric sensor/actuator network: I. diagnostics. Smart Mater Struct 2004; 13(3): 609.

[4] Ihn JB and Chang FK. Detection and monitoring of hidden fatigue crack growth using a built-in piezoelectric sensor/actuator network: II. Validation using riveted joints and repair patches. Smart Mater Struct 2004; 13(3): 621.

[5] Su Z, Ye L and Lu Y. Guided lamb waves for identification of damage in composite structures: A review. J Sound Vibration 2006; 295(3): 753-780.

[6] Xu B and Giurgiutiu V. Single mode tuning effects on Lamb wave time reversal with piezoelectric wafer active sensors for structural health monitoring. J Nondestruct Eval 2007; 26(2): $123-134$.

[7] Yu L and Giurgiutiu V. In situ 2-D piezoelectric wafer active sensors arrays for guided wave damage detection. Ultrasonics 2008; 48(2): 117-134.

[8] Michaels JE. Detection, localization and characterization of damage in plates with an in situ array of spatially distributed ultrasonic sensors. Smart Mater Struct 2008; 17(3): 035035.

[9] Quaegebeur N, Masson P, Langlois-Demers D et al. Dispersion-based imaging for structural health monitoring using sparse and compact arrays. Smart Mater Struct 2011; 20(2): 025005.

[10] Qiu L, Yuan S, Zhang X et al. A time reversal focusing based impact imaging method and its evaluation on complex composite structures. Smart Mater Struct 2011; 20(10): 105014.

[11] De Marchi L, Marzani A, Speciale N et al. A passive monitoring technique based on dispersion compensation to locate impacts in plate-like structures. Smart Mater Struct 2011; 20(3): 035021.

[12] Quaegebeur N, Ostiguy P and Masson P. Correlation-based imaging technique for fatigue monitoring of riveted lap-joint structure. Smart Mater Struct 2014; 23(5): 055007.

[13] Levine R and Michaels JE. Block-sparse reconstruction and imaging for lamb wave structural health monitoring. IEEE T Ultrason Ferr 2014; 61(6): 1006-1015.

[14] Hall JS and Michaels JE. Multipath ultrasonic guided wave imaging in complex structures. Struct Health Monit 2015; 14(4): 345-358.

[15] Moriot J, Maxit L, Guyader JL et al. Use of beamforming for detecting an acoustic source inside a cylindrical shell filled with a heavy fluid. Mech Syst Signal Process 2015; 52: 645-662. 
[16] Ostiguy PC, Quaegebeur N and Masson P. Comparison of model-based damage imaging techniques for transversely isotropic composites. Struct Health Monit 2016; : 1475921716674012.

[17] Georgiou G. Probability of detection (PoD) curves. derivation, application and limitations. Technical report, Jacobi Consulting Limited, 2006.

[18] Department of Defense Handbook: Nondestructive Evaluation System Reliability Assessment. MIL-HDBK- 1823A. Technical report, 2009.

[19] Janapati V, Kopsaftopoulos F, Li F et al. Damage detection sensitivity characterization of acousto-ultrasound-based structural health monitoring techniques. Struct Health Monit 2016; 15(2): $143-161$.

[20] Ihn JB and Chang FK. Pitch-catch active sensing methods in structural health monitoring for aircraft structures. Struct Health Monit 2008; 7(1): 5-19.

[21] Thompson RB, Thompson DO and Chimenti DE. A Unified Approach To the Model-Assisted Determination of Probability of Detection. AIP Conference Proceedings 2008; 975(1): 16851692.

[22] Kulkarni S and Achenbach J. Structural health monitoring and damage prognosis in fatigue. Struct Health Monit 2008; 7(1): 37-49.

[23] Cobb AC, Michaels JE, Michaels TE et al. Ultrasonic structural health monitoring: a probability of detection case study. AIP Conference Proceedings 2009; 1096(1): 1800-1807.

[24] Lu Y and Michaels JE. Feature extraction and sensor fusion for ultrasonic structural health monitoring under changing environmental conditions. IEEE Sens J 2009; 9(11): 1462-1471.

[25] Aldrin JC, Medina EA, Lindgren EA et al. Model-assisted probabilistic reliability assessment for structural health monitoring systems. AIP Conference Proceedings 2010; 1211(1): 19651972.

[26] Mueller I, Janapati V, Banerjee S et al. On the performance quantification of active sensing SHM systems using model-assisted POD methods. in: Proceedings of the 8th international workshop on structural health monitoring, Stanford CA, USA, 2011, pp.2417-2428 ; .

[27] Flynn EB, Todd MD, Wilcox PD et al. Maximum-likelihood estimation of damage location in guided-wave structural health monitoring. Proc R Soc A 2011; : 20110095.

[28] Flynn EB, Todd MD, Croxford AJ et al. Enhanced detection through low-order stochastic modeling for guided-wave structural health monitoring. Struct Health Monit 2012; 11(2): 149 160.

[29] Jarmer GJ, Flynn EB and Todd MD. Multi-wave-mode, multi-frequency detectors for guided wave interrogation of plate structures. Struct Health Monit 2014; 13(2): 120-130.

[30] Sharif Khodaei Z and Aliabadi M. Assessment of delay-and-sum algorithms for damage detection in aluminium and composite plates. Smart Mater Struct 2014; 23(7): 075007. 
[31] Fendzi C, Morel J, Rebillat M et al. Optimal sensors placement to enhance damage detection in composite plates. In: Proceedings of the 7th European Workshop on Structural Health Monitoring, Nantes, France, 2014, pp.1-8 ; .

[32] Roach D, Rice T, Neidigk S et al. Establishing the Reliability of SHM Systems Through the Extrapolation of NDI Probability of Detection Principles. in: Proceedings of the international workshop on structural health monitoring, Stanford CA, USA, 2015 ; .

[33] Jarmer G and Kessler S. Probability of detection assessment of a guided wave structural health monitoring system. in: Proceedings of the international workshop on structural health monitoring, Stanford CA, USA, 2015.

[34] Schubert Kabban CM, Greenwell BM, DeSimio MP et al. The probability of detection for structural health monitoring systems: Repeated measures data. Struct Health Monit 2015; 14(3): 252-264.

[35] Aldrin JC, Annis C, Sabbagh HA et al. Best practices for evaluating the capability of nondestructive evaluation (NDE) and structural health monitoring (SHM) techniques for damage characterization. AIP Conference Proceedings 2016; 1706(1): 200002.

[36] Gianneo A, Carboni M and Giglio M. Feasibility study of a multi-parameter probability of detection formulation for a lamb waves-based structural health monitoring approach to light alloy aeronautical plates. Struct Health Monit 2016; : 1475921716670841.

[37] Memmolo V, Ricci F, Maio L et al. Model assisted probability of detection for a guided-waves based SHM technique. In: Proceedings of SPIE 9805, Health Monitoring of Structural and Biological Systems, Las Vegas, NV, USA, 2016, pp.980504-980504; .

[38] Hall JS and Michaels JE. Minimum variance ultrasonic imaging applied to an in situ sparse guided wave array. IEEE T Ultrason Ferr 2010; 57(10).

[39] Michaels JE, Jacobs LJ and Ruzzene M. A Multi-Scale Structural Health Monitoring Approach for Damage Detection, Diagnosis and Prognosis in Aerospace Structures. Technical report, DTIC Document, 2012.

[40] Qiu L, Liu M, Qing X et al. A quantitative multidamage monitoring method for large-scale complex composite. Struct Health Monit 2013; 12(3): 183-196.

[41] Kopsaftopoulos F and Fassois S. A functional model based statistical time series method for vibration based damage detection, localization, and magnitude estimation. Mech Syst Signal Process 2013; 39(1): 143-161.

[42] Mallardo V, Aliabadi M and Sharif-Khodaei Z. Optimal sensor positioning for impact localization in smart composite panels. J Intell Mater Syst 2013; 24(5): 559-573.

[43] Sharif Khodaei Z and Aliabadi M. A Multi-Level Decision Fusion Strategy for Condition Based Maintenance of Composite Structures. Materials 2016; 9(9): 790.

[44] Moriot J, Quaegebeur N, Le Duff A et al. Characterization of the robustness of SHM imaging techniques using the absolute error of localization. In: Proceedings of the 8th European Workshop on Structural Health Monitoring, Bilbao, Spain, 2016 ; . 
[45] Annis C, Knopp J, Thompson DO et al. Comparing the effectiveness of a90/95 calculations. AIP Conference Proceedings 2007; 894(1): 1767-1774.

[46] Levine RM and Michaels JE. Model-based imaging of damage with Lamb waves via sparse reconstruction. J Acoust Soc Am 2013; 133(3): 1525-1534.

[47] Quaegebeur N, Padois T, Gauthier PA et al. Enhancement of time-domain acoustic imaging based on generalized cross-correlation and spatial weighting. Mech Syst Signal Process 2016; 75: $515-524$.

[48] Wilcox PD. A rapid signal processing technique to remove the effect of dispersion from guided wave signals. IEEE T Ultrason Ferr 2003; 50(4): 419-427.

[49] Raghavan A and Cesnik CE. Finite-dimensional piezoelectric transducer modeling for guided wave based structural health monitoring. Smart Mater Struct 2005; 14(6): 1448.

[50] Sohn H and Lee SJ. Lamb wave tuning curve calibration for surface-bonded piezoelectric transducers. Smart Mater Struct 2009; 19(1): 015007.

[51] Yeasin Bhuiyan M, Shen Y and Giurgiutiu V. Interaction of Lamb waves with rivet hole cracks from multiple directions. Proc Inst Mech Eng C J Mech Eng Sci 2017; : 0954406216686996.

[52] Quaegebeur N, Bouslama N, Bilodeau M et al. Guided wave scattering by geometrical change or damage: Application to characterization of fatigue crack and machined notch. Ultrasonics 2017; 73: 187-195.

[53] Chen X, Michaels JE and Michaels TE. A methodology for estimating guided wave scattering patterns from transducer array measurements. IEEE T Ultrason Ferr 2015; 62(1): 208-219.

[54] Norris A and Vemula C. Scattering of flexural waves on thin plates. J Sound Vibration 1995; 181(1): $115-125$.

[55] Sakia R. The Box-Cox Transformation Technique: A Review. J Royal Stat Soc Series D (The Statistician) 1992; 41(2): 169-178.

[56] McZgee VE and Carleton WT. Piecewise Regression. J Am Stat Assoc 1970; 65(331): 11091124.

[57] Spencer FW. Nonparametric POD Estimation for Hit/Miss Data: A Goodness of Fit Comparison for Parametric Models. In AIP Conference Proceedings, volume 1335. AIP, pp. 1557-1564. 